Published in "Physica A: Statistical Mechanics and its Applications 402: 180-185, 2014"

which should be cited to refer to this work.

\title{
Network skeleton for synchronization: Identifying redundant connections
}

\author{
Cheng-Jun Zhang, An Zeng* \\ Institute of Information Economy, Alibaba Business School, Hangzhou Normal University, Hangzhou, 310036, PR China \\ Department of Physics, University of Fribourg, Chemin du Musée 3, CH-1700 Fribourg, Switzerland
}

\section{H I G H L I G H T S}

- We find that many links in networks are actually redundant for synchronization.

- The homogeneous networks have more redundant links than the heterogeneous networks.

- The synchronization backbone is the minimal network to preserve synchronizability.

- The results are confirmed by the Kuramoto model.

\begin{abstract}
Synchronization is an important dynamical process on complex networks with wide applications. In this paper, we design a greedy link removal algorithm and find that many links in networks are actually redundant for synchronization, i.e. the synchronizability of the network is hardly affected if these links are removed. Our analysis shows that homogeneous networks generally have more redundant links than heterogeneous networks. We denote the reduced network with the minimum number of links to preserve synchronizability (eigenratio of the Laplacian matrix) of the original network as the synchronization backbone. Simulating the Kuramoto model, we confirm that the network synchronizability is effectively preserved in the backbone. Moreover, the topological properties of the original network and backbone are compared in detail.
\end{abstract}

\section{Introduction}

Understanding the relation between network structure and dynamical processes is one of the most significant topics in network researches [1]. Synchronization is a typical collective process in many different fields including biology, physics, engineering, and even sociology. It is known that synchronization is rooted in human life from the metabolic processes in our cells to the highest cognitive tasks we perform as a group of individuals. Since the small-world and scale-free networks were proposed, the synchronization on complex networks has been intensively investigated [2-7]. Many of these studies are based on the analysis of Master Stability Function which allows us to use the eigenratio $R=\lambda_{2} / \lambda_{N}$ of the Laplacian matrix to represent the synchronizability of a network $[8,9]$. The larger the eigenratio is, the stronger the synchronizability of the network is.

In the literature, many works focus on investigating how the network structure affects the synchronizability [10-14]. Since the network synchronizability can be significantly influenced by network structure, researchers proposed many methods to modify the topology of networks to enhance the synchronizability. For instance, Refs. $[15,16]$ improve the synchronizability by adding, removing and rewiring links in networks. The synchronizability is also enhanced by manipulating links

\footnotetext{
* Corresponding author at: Department of Physics, University of Fribourg, Chemin du Musée 3, CH-1700 Fribourg, Switzerland. Tel.: +41 0263009275. E-mail address: an.zeng@unifr.ch (A. Zeng).
} 
based on evolutionary optimization algorithms [17-19]. Additionally, the synchronizability can be improved by properly assigning link directions $[9,20]$. Weighting links is another way to improve the network synchronizability [3,21,22]. For instance, researchers design some strategies to make the strength of nodes homogeneous and reduce the feedback loop [2]. Through these link weighting methods, it is finally found that the directed spanning tree for any connected initial network configuration can achieve the strongest synchronizability (i.e., $\lambda_{2}=\lambda_{N}=1$ and $R=1$ ) [23,24]. In Ref. [25], it is further demonstrated that spanning trees are not the only optimal networks for synchronization. Very recently, it is revealed that the transition from disorder state to synchronization can be the first order if the natural frequency of the oscillators are positively correlated with node degree [26].

Instead of improving the network synchronizability, we focus in this paper on investigating the redundant links for synchronization. The network synchronizability will stay almost unchanged if these links are removed. In order to accurately identify and remove the redundant links, we design a greedy algorithm based on the eigenvector of the Laplacian matrix. We validate the algorithm by directly comparing the simulation of the Kuramoto model on the original network and the reduced network [27]. With this algorithm, we find that the homogeneous networks generally have more redundant links than the heterogeneous networks do.

The above phenomenon indicates that there is a backbone structure for the network synchronization. Generally, a backbone should preserve the topological properties or the function of the original networks. For example, the degree distribution [28], betweenness [29] and transportation ability [30] and even the ability for information filtering [31] can be preserved in network backbones. More recently, a network backbone detection method based on link salience is proposed [32]. In this paper, we remove the redundant links until the synchronizability is reduced to $95 \%$ of the original one. The obtained network is considered as the synchronization backbone. The topological properties of the original network and backbone are compared in detail. Finally, we remark that this work is also meaningful from a practical point of view. Since the backbone has almost the same synchronizability of the original network but has $30 \%$ fewer links, the construction cost of real systems can be considerably reduced by excluding the links outside the backbone structure.

\section{Redundant links for synchronizability in complex networks}

In a dynamical network, each node represents an oscillator and the edges represent the couplings between the nodes. For a network of $N$ linearly coupled identical oscillators, the dynamical equation of each oscillator can be written as

$$
\dot{x}_{i}=F\left(x_{i}\right)-\sigma \sum_{j=1}^{N} G_{i j} H\left(x_{j}\right), \quad i=1,2, \ldots, N .
$$

where $x$ is the phase of each oscillator, $F(x)$ accounts for the internal dynamics of each node, $H(x)$ is a coupling function, and $\sigma$ is the coupling strength. The previous works based on the analysis of Master Stability Function show that the network synchronizability can be measured by eigenratio $R=\lambda_{2} / \lambda_{N}$ where $\lambda_{2}$ and $\lambda_{N}$ are respectively the smallest nonzero and largest eigenvalues of the Laplacian matrix [8].

By defining the eigenvector corresponding to the largest eigenvalue as $v_{N}$, we can get the following formula

$$
\lambda_{N}=\sum_{i \sim j}\left(v_{N}(i)-v_{N}(j)\right)^{2},
$$

subject to $\sum_{i} v_{N}(i)^{2}=1$ [33]. This suggests that $\lambda_{N}$ will stay unchanged if we remove the edge $(i, j)$ which minimizes $\left|v_{N}(i)-v_{N}(j)\right|[15]$. Actually, the same ideas work for the smallest nonzero eigenvalue $\lambda_{2}$ and associated eigenvector $v_{2}$. The analysis indicates that not every link contributes to the network synchronizability. We call these links as the redundant links for synchronization. In the next section, we will design algorithms to identify and delete the redundant links from networks.

\section{The greedy algorithms to remove redundant links}

We first denote the synchronizability of the original network as $R^{(0)}$. After removing $l$ links, the synchronizability of the reduced network is denoted as $R^{(l)}$. Naturally, the most straightforward way to design the greedy algorithm is to remove the link which minimizes

$$
\Delta R=\left|R^{(l)}-R^{(l-1)}\right| .
$$

In practice, we calculate $\Delta R$ of each remaining link (by assuming this link is removed) and select the link with lowest $\Delta R$ to be actually removed from the network. With this method, one can effectively preserve the $R$ value even when a considerable number of links are cut from the network. For convenience, this method is called Greedy Algorithm based on $R$ (for short GAR).

We test the above algorithm in two kinds of modeled networks: (i) the Watts-Strogatz network (WS network) [34]: the model starts from a completely regular network with identical degree. Each link will be rewired with two randomly selected nodes with probability $q \in(0,1)$. (ii) the Barabasi-Albert network (BA network) [35]: starting from $m$ all to all connected nodes, at each time step a new node is added with $m$ links. These $m$ links connect to old nodes with probability $p_{i}=k_{i} / \sum_{j} k_{j}$, where $k_{i}$ is the degree of the node $i$. Besides this standard BA model, we will consider later a variant of it 

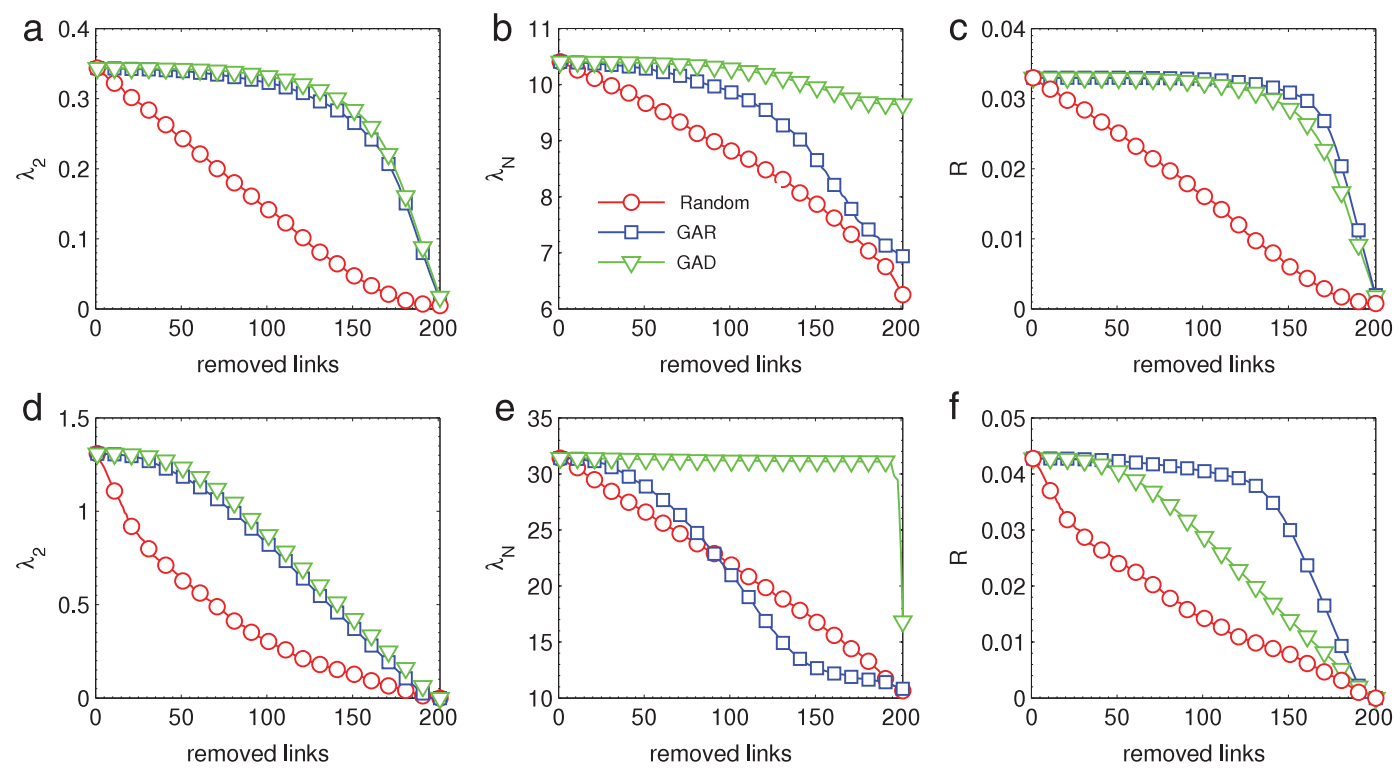

Fig. 1. (Color online) The dependence of $\lambda_{2}, \lambda_{N}$ and eigenratio $R$ on the removed links in (a)-(c) WS network $[N=100, k=6, p=0.1]$ and (d)-(f) BA network $[N=100, k=6]$, respectively. The results are averaged over 100 independent realizations.

where $p_{i}=\left(k_{i}+B\right) / \sum_{j}\left(k_{j}+B\right)$ [22]. This modified model allows a selection of the exponent of the power-law scaling in the degree distribution $p(k) \sim k^{-\gamma}$, with $\gamma=3+B / m$ in the thermodynamic limit. The results of GAR on the WS and BA models are reported in Fig. 1(c) and (f). One can see that $R$ can stay almost unchanged even when $50 \%$ of the links are removed from the WS network and 30\% links are removed from the BA network. On the other hand, $R$ changes dramatically when links are removed randomly.

In fact, simply preserving $R$ value is not enough to keep the synchronization unchanged [36,37]. According to the analysis in Section 2, the network synchronizability is determined by both $\lambda_{N}$ and $\lambda_{2}$. It is also shown in Fig. 1 that $\lambda_{N}$ decreases as fast as the random link removal if GAR is applied. In order to achieve a better preservation of the synchronizability, we propose an improved greedy algorithm which takes both $\lambda_{2}$ and $\lambda_{N}$ into account. In each step, we calculate the value of $\left|\lambda_{2}^{(l)}-\lambda_{2}^{(l-1)}\right|$ and $\left|\lambda_{N}^{(l)}-\lambda_{N}^{(l-1)}\right|$ of each testing link and select the link with the minimal

$$
\Delta D=\frac{\left|\lambda_{2}^{(l)}-\lambda_{2}^{(l-1)}\right|}{\lambda_{2}^{(l-1)}}+\frac{\left|\lambda_{N}^{(l)}-\lambda_{N}^{(l-1)}\right|}{\lambda_{N}^{(l-1)}}
$$

to be really removed from the network. This method is called the Greedy Algorithm based on $D$ (for short GAD). The results of GAD are presented in Fig. 1 as well. Even though $R$ of the GAD decays faster than that of GAR, both $\lambda_{2}$ and $\lambda_{N}$ are better preserved in GAD.

We remove links by GAD until $R^{(l)}$ is lower than $95 \%$ of $R^{(0)}$ and the obtained network is denoted as the synchronization backbone. Since we remove links one by one, it is necessary to check whether the results strongly depend on the order of link removal. After getting the synchronization backbone, we keep record of all the removed links. Then we start from the original network again and remove these links in random order. It can be seen clearly from Fig. 2 that the order of link removal does not significantly influence the $R$ value (the error bars are relatively small). This result confirms that all these links are indeed redundant for the network synchronizability.

In Fig. 2, it is also easy to notice that the number of removable links (redundant links) is different in two network models. It is interesting to investigate how the network topology affects the removable links. Firstly, we study the relation between the average degree of the network and ratio of removable links. As shown in Fig. 3, the ratio of removable links increases with the average degree in both network models and stay stable when $\langle k\rangle$ is large. The ratio of removable links in the WS network reaches the stable state earlier than that in the BA network. Moreover, the ratio of removable links is generally higher in the WS network. We further study the rewiring parameter $p$ in the WS network and the exponent adjusting parameter $B$ in the modified BA model. Interestingly, the fraction of removable links first increases then decreases with $p$. It indicates that the small-world network only needs a small number of essential links to keep its synchronizability. However, the removable links become significantly fewer when the WS model degenerates to the regular network $(p=0)$ and the random network $(p=1)$. In the modified BA model, we observe that the fraction of removable links keeps increasing with $B$. In this network model, the heterogeneity of the degree distribution can be adjusted by $B$. A larger $B$ corresponds to a network with more homogeneous degree distribution. The results indicate that the network with heterogeneous degree distribution needs more links to keep its synchronizability. 

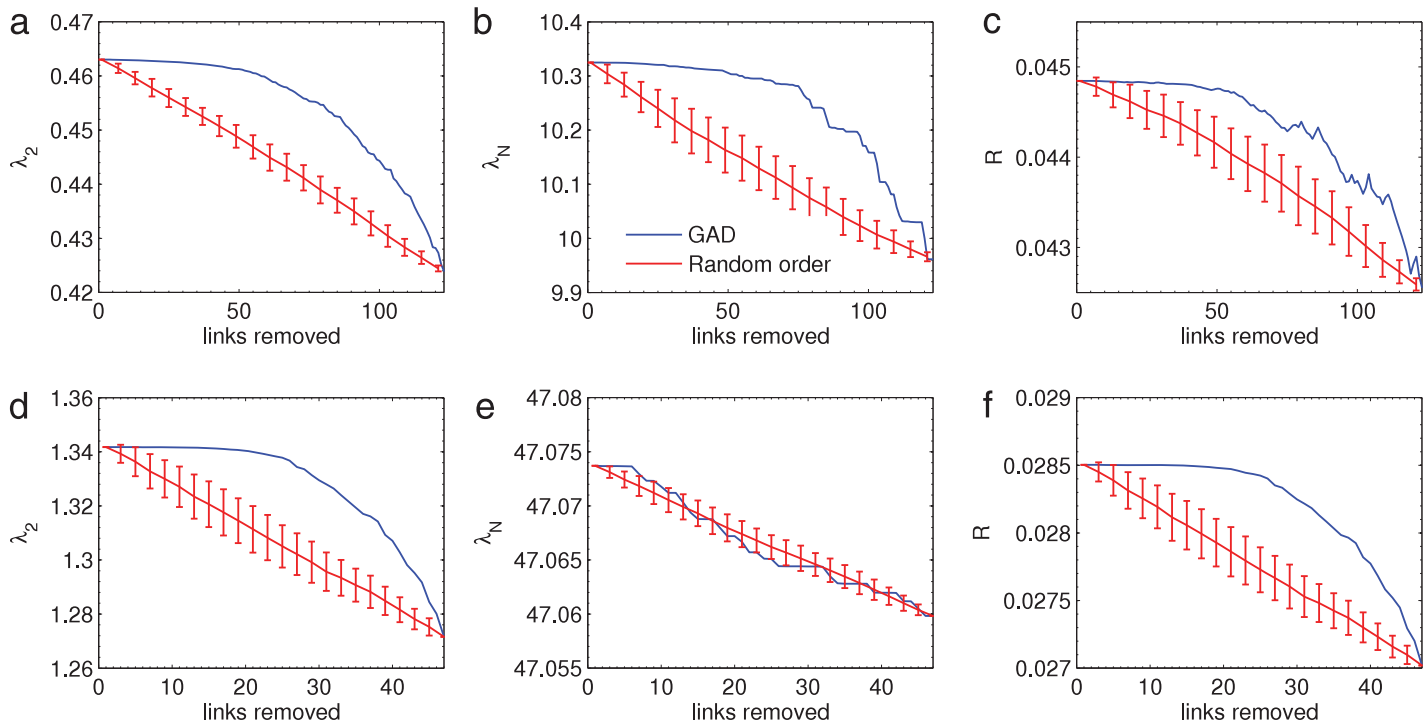

Fig. 2. (Color online) Removing the redundant links based on randomized order in (a)-(c) WS network and (d)-(f) BA network. The network parameters are the same as those in Fig. 1 . The results are averaged over 100 independent realizations.
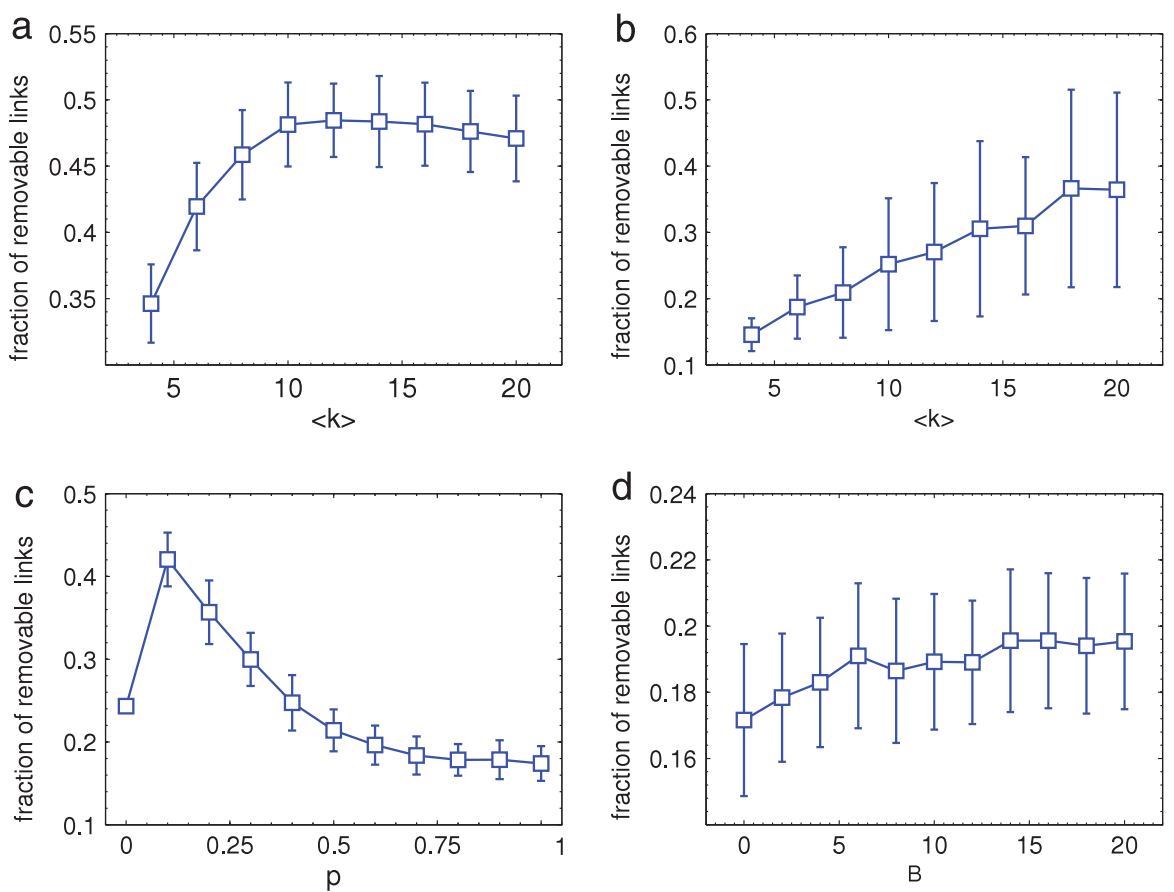

Fig. 3. (Color online) The dependence of the redundant link rate on average degree in (a) WS network $[N=100, p=0.1]$ and (b) BA network $[N=100]$. (c) is the relation between the redundant link rate and the rewiring probability $p$ in WS network $[N=100, k=6]$. (d) is the relation between the redundant link rate and the parameter $B$ in the variant of the BA model $[N=100, k=6]$. The results are averaged over 10 independent realizations.

Next, we investigate the structural features of the obtained synchronization backbone. We compare the original networks and the backbones in five structure indices including the maximum degree $k_{\max }$, degree heterogeneity $H$, clustering coefficient $\langle C\rangle$ [34], assortativity $r$ [38] and average shortest path length $\langle d\rangle$ [34]. The results of the above structure indices are reported in Table 1. Different from the random link removal, $k_{\max }$ is well preserved in the backbone structure. This is because $k_{\max }$ is closely related to $\lambda_{N}$, and $\lambda_{N}$ needs to be kept almost unchanged in the backbone. Since these large degree nodes are preserved while the links between the small degree nodes are removed, the assortativity $r$ in the backbone is slightly increased. Moreover, the clustering coefficient of the backbone is significantly smaller than that from the random link removal. This indicates that the redundant links are mainly located in the local region. Since many links are removed, 
Table 1

The topology properties (the total number of links $E$, the maximum degree $k_{\max }$, the degree heterogeneity $H$, the clustering coefficient $\langle C\rangle$, the assortativity $r$ and the average shortest path length $\langle d\rangle$ ) in different networks. All networks are with $N=100$ and the results are averaged over 100 independent realizations.

\begin{tabular}{|c|c|c|c|c|c|c|c|}
\hline Method & E & $k_{\max }$ & $H$ & $\langle C\rangle$ & $r$ & $\langle d\rangle$ & Net. \\
\hline Original & 300 & 8.17 & 1.016 & 0.448 & -0.023 & 3.636 & \\
\hline Backbone & 175.16 & 8.15 & 1.184 & 0.078 & -0.039 & 4.139 & WS \\
\hline Random & 175.16 & 6.41 & 1.128 & 0.245 & -0.029 & 5.317 & \\
\hline Original & 291 & 30.04 & 1.670 & 0.149 & -0.152 & 2.593 & \\
\hline Backbone & 237.53 & 30.04 & 1.721 & 0.098 & -0.188 & 2.759 & BA \\
\hline Random & 237.53 & 25.00 & 1.703 & 0.114 & -0.149 & 2.871 & \\
\hline Original & 300 & 11.15 & 1.080 & 0.051 & -0.071 & 2.729 & \\
\hline Backbone & 246.46 & 11.12 & 1.097 & 0.017 & -0.134 & 2.959 & ER \\
\hline Random & 246.46 & 9.81 & 1.116 & 0.042 & -0.058 & 3.037 & \\
\hline
\end{tabular}

the average shortest path length is inevitably increased. However, one can see that the average shortest path length of the backbone is closer to that of the original network. This is because the average shortest path length is one of the most important network properties to determine the synchronizability [2]. In order to preserve the synchronizability, it is necessary for the backbone to keep the shortest path length close to that of the original network.

\section{The Kuramoto model}

For the purpose of comparing the actually synchronization process on the original network and the backbone, we study the Kuramoto model in both networks. The Kuramoto model is a classical model to investigate the phase synchronization phenomenon [27]. The coupled Kuramoto model in the network can be written as [9]

$$
\dot{\theta}_{i}=\omega_{i}+K / \bar{k} \sum_{j}^{N} a_{i j} \sin \left(\theta_{j}-\theta_{i}\right), \quad i=1,2, \ldots, N,
$$

where $\theta_{i}$ and $\omega_{i}$ are the phase and natural frequency of the oscillator $i$, respectively. $K$ is the coupling strength and $\bar{k}$ is the average degree of the network.

The collective dynamics of the whole population at step $t$ is measured by the macroscopic complex order parameter,

$$
r(t) \mathrm{e}^{\mathrm{i} \phi(t)}=\frac{1}{N} \sum_{j=1}^{N} \mathrm{e}^{\mathrm{i} \theta_{j}(t)},
$$

where $r(t) \simeq 1$ and $r(t) \simeq 0$ describe the limits in which all oscillators are either phase locked or moving incoherently, respectively. Here, we denote $r$ as the value of $r(t)$ when $t$ is infinitely large (i.e. when $r(t)$ reaches stable state).

We first use GAD to remove $l$ links from the WS and BA networks until $R^{(l)}$ decreases to $95 \%$ of $R^{(0)}$ (the synchronization backbone). We then use GAR and the random method to remove the same number of links from the same original WS and BA networks. In Fig. 4(a) and (b), we investigate the behavior of $r$ vs $K$ on the WS, BA and their reduced networks after link removal. From Fig. 1(c) and (f), we can see that $R_{\mathrm{GAR}}^{(l)}$ is closer to $R^{(0)}$ than $R_{\mathrm{GAD}}^{(l)}$ when the same number of links are removed. However, when simulating the Kuramoto model, we find that the curves of $r$ in GAD overlap better to the $r$ curve of the original network than that of GAR. This result implies that a good algorithm should preserve both $\lambda_{N}$ and $\lambda_{2}$ (not just the eigenratio). To further confirm the results, we plot the difference of Kuramoto's $r$ between the reduced networks and the original network $\left(\Delta r=r^{(l)}-r^{(0)}\right.$ where $r^{(l)}$ and $r^{(0)}$ are the order parameters of the reduced networks and original network, respectively). As one can see in Fig. 4(c) and (d), the reduced network from GAD indeed has the smallest difference to the original network.

\section{Conclusion}

Synchronization on complex networks has been intensively studied in the past decade. So far, much effort has been devoted to enhance network synchronizability. Different from previous works, we focus in this paper on identifying the redundant links for synchronization in complex networks, i.e., the synchronizability will be preserved if these links are removed. We design a greedy algorithm to remove the redundant links and obtain the backbone for synchronization. The result is confirmed by the numerical simulation of the Kuramoto model.

In reality, a network may have more than one function. For example, the distributed power generation systems have to synchronize the injected current into the utility network with the grid voltage. Meanwhile, it should be robust to some unexpected power station failures or malicious attacks. Removing the redundant connections for network synchronizability may significantly change the network robustness. Therefore, how to identify the redundant links for multiple network functions is still an open issue which calls for future investigation. 

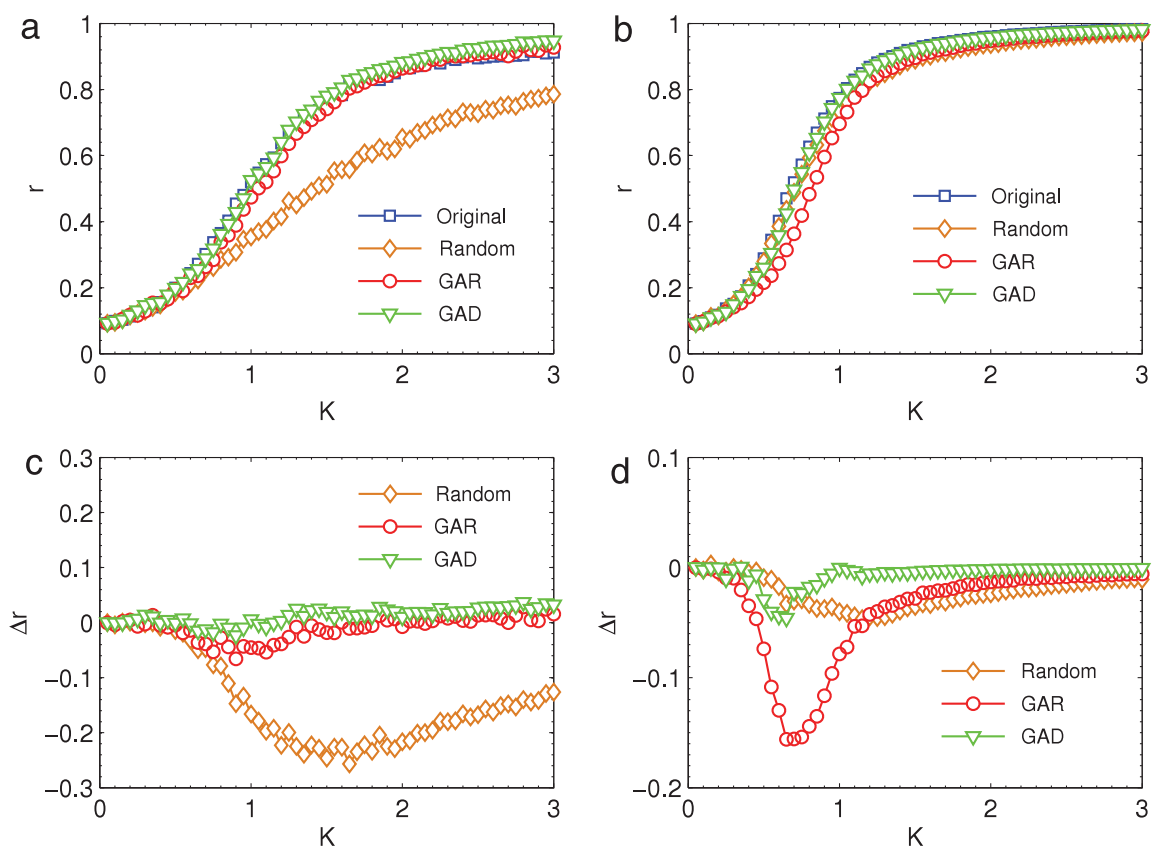

Fig. 4. (Color online) Dynamic behavior of the Kuramoto model in the obtained network from different link removal methods in (a) WS and (b) BA networks. The difference between $r$ in the obtained networks and original network. The original networks are (a) WS networks and (b) BA networks, respectively. The network parameters are the same as in Fig. 1. The results are averaged over 50 independent realizations.

\section{Acknowledgments}

This work is supported by the opening foundation of Institute of Information Economy in Hangzhou Normal University (Grant No. PD12001003002002). The authors also acknowledge the support from the China Scholarship Council.

\section{References}

11 S. Boccaletti, V. Latora, Y. Moreno, M. Chavez, D.-U. Hwang, Phys. Rep. 424 (2006) 175.

$2]$ A. Arenas, A. Diaz-Guilera, J. Kurths, Y. Moreno, C.-S. Zhou, Phys. Rep. 469 (2008) 93.

A.E. Motter, C. Zhou, J. Kurths, Phys. Rev. E 71 (2005) 016116.

J.-G. Liu, T. Zhou, Q. Guo, B.-H. Wang, Internat. J. Modern Phys. C 19 (9) (2008) 1359

5] Q. Guo, J.-G. Liu, R.-L. Wang, X.-W. Chen, Y.-H. Yao, Chin. Phys. Lett. 24 (8) (2007) 2437.

6] A. Zeng, Y. Hu, Z. Di, Europhys. Lett. 87 (2009) 48002.

T. Zhou, M. Zhao, C. Zhou, New J. Phys. 12 (2010) 043030.
M. Barahona, L.M. Pecora, Phys. Rev. Lett. 89 (2002) 054101

S.-W. Son, B.J. Kim, H. Hong, H. Jeong, Phys. Rev. Lett. 103 (2009) 228702.

10] T. Nishikawa, A.E. Motter, Y.-C. Lai, F.C. Hoppensteadt, Phys. Rev. Lett. 91 (2003) 014101.

11] H. Hong, B.J. Kim, M.Y. Choi, H. Park, Phys. Rev. E 69 (2004) 067105.

12] P.N. McGraw, M. Menzinger, Phys. Rev. E 72 (2005) 015101(R)

$13]$ M. Zhao, T. Zhou, B.-H. Wang, G. Yan, H.-J. Yang, W.-J. Bai, Physica A 371 (2006) 773.

14 W.X. Wang, T. Zhou, W.-X. Wang, M. Zhao, H.-J. Yang, Chin. Phys. Lett. 23 (2006) 1046

15] A. Hagberg, D.A. Schult, Chaos 18 (2008) 037105.

16] A. Zeng, L. Lü, T. Zhou, New J. Phys. 14 (2012) 083006

17] L. Donetti, P.I. Hurtado, M.A. Munoz, Phys. Rev. Lett. 95 (2005) 188701.

18] M. Brede, Eur. Phys. J. B 74 (2010) 217.

19] M. Jalili, A.A. Rad, Chaos 19 (2009) 028101.

20] A. Zeng, S.-W. Son, C.H. Yeung, Y. Fan, Z. Di, Phys. Rev. E 83 (2011) 045101(R)

21] C. Zhou, A.E. Motter, J. Kurths, Phys. Rev. Lett. 96 (2006) 034101.

[22] M. Chavez, D.-U. Hwang, A. Amann, H.G.E. Hentschel, S. Boccaletti, Phys. Rev. Lett. 94 (2005) 218701.

$23]$ T. Nishikawa, A.E. Motter, Phys. Rev. E 73 (2006) 065106.

24] T. Nishikawa, A.E. Motter, Physica D 224 (2006) 77

[25] T. Nishikawa, A.E. Motter, Proc. Natl. Acad. Sci. USA 107 (2010) 10342

26] J. Gomez-Gardenes, S. Gomez, A. Arenas, Y. Moreno, Phys. Rev. Lett. 106 (2011) 128701.

27] Y. Kuramoto, Lecture Notes in Phys. 39 (1975) 420.

28] M.A. Serrano, M. Boguna, A. Vespignani, Proc. Natl. Acad. Sci. USA 106 (2009) 6483.

29] D.H. Kim, J.D. Noh, H. Jeong, Phys. Rev. E 70 (2004) 046126.

30] Z. Wu, L.A. Braunstein, S. Havlin, H.E. Stanley, Phys. Rev. Lett. 96 (2006) 148702.

1 Q.-M. Zhang, A. Zeng, M.-S. Shang, PLoS One 8 (5) (2013) e62624.

32] D. Grady, C. Thiemann, D. Brockmann, Nature Commun. 3 (2013) 864

33] R. Merris, Linear Algebra Appl. 198 (1994) 143.

[34] D.J. Watts, S.H. Strogatz, Nature 393 (1998) 440.

35] A.-L. Barabasi, R. Albert, Science 286 (1999) 509.

36] D. Gfeller, P. De Los Rios, Phys. Rev. Lett. 100 (2008) 174104

37] A. Zeng, L. Lü, Phys. Rev. E E 83 (2011) 056123.

[38] M.E.J. Newman, Phys. Rev. Lett. 89 (2002) 208701. 